\title{
Asymmetric interlimb transfer of concurrent adaptation to opposing dynamic forces
}

\author{
J. M. Galea · R. C. Miall · D. G. Woolley
}

Received: 4 April 2007/Accepted: 15 July 2007/Published online: 17 August 2007

(C) Springer-Verlag 2007

\begin{abstract}
Interlimb transfer of a novel dynamic force has been well documented. It has also been shown that unimanual adaptation to opposing novel environments is possible if they are associated with different workspaces. The main aim of this study was to test if adaptation to opposing velocity dependent viscous forces with one arm could improve the initial performance of the other arm. The study also examined whether this interlimb transfer occurred across an extrinsic, spatial, coordinative system or an intrinsic, joint based, coordinative system. Subjects initially adapted to opposing viscous forces separated by target location. Our measure of performance was the correlation between the speed profiles of each movement within a force condition and an 'average' trajectory within null force conditions. Adaptation to the opposing forces was seen during initial acquisition with a significantly improved coefficient in epoch eight compared to epoch one. We then tested interlimb transfer from the dominant to non-dominant arm $(\mathrm{D} \rightarrow \mathrm{ND})$ and vice-versa $(\mathrm{ND} \rightarrow \mathrm{D})$ across either an extrinsic or intrinsic coordinative system. Interlimb transfer was only seen from the dominant to the non-dominant limb across an intrinsic coordinative system. These results support previous studies involving adaptation to a single dynamic force but also indicate that interlimb transfer of multiple opposing states is possible. This suggests that the information available at the level of
\end{abstract}

J. M. Galea $(\bowtie) \cdot$ R. C. Miall

Behavioural Brain Sciences Centre,

School of Psychology, University of Birmingham,

Edgbaston, Birmingham, West Midlands B15 2TT, UK

e-mail: jmg111@bham.ac.uk

D. G. Woolley

Perception and Motor Systems Laboratory,

The University of Queensland, Brisbane, QLD 4072, Australia representation allowing interlimb transfer can be more intricate than a general movement goal or a single perceived directional error.

\section{Introduction}

It has recently been shown that if a subject learns to adapt to a novel environment with one arm it improves the subsequent performance of the other (CriscimagnaHemminger et al. 2003; Dizio and Lackner, 1995; Malfait and Ostry 2004; Sainburg and Wang 2002; Wang and Sainburg 2003, 2004a, b) The observed interlimb transfer is not symmetrical, with the direction of transfer dependent on the type of novel environment and on the movement parameter being measured.

Hwang et al. (2006) have recently proposed that two internal models are formed during adaptation to novel dynamics; one mainly based on proprioception and the other on vision. During dynamic adaptation the proprioceptive model strongly influences reaching performance but does not affect awareness of the adaptive state. The visual model strongly influences awareness but has a much smaller effect on reaching performance. The authors believe that performance is the sum of a strong implicit model that is based on proprioception and is effector dependent and a weak explicit model that depends on vision and is effector independent. Specifically they predict that interlimb transfer is a consequence of the subject becoming explicitly aware of the novel environment.

The situations in which interlimb transfer has been reported have largely been restricted to adaptation to a single kinematic or dynamic novel environment. It is unclear whether transfer would persist when encountering a 
more complicated task. Wang and Sainburg (2004b) have shown that, unlike intralimb adaptation, movements to multiple target directions does not improve generalisation across limbs. They believe that interlimb transfer occurs upstream from the effector-specific generalisation seen in multiple direction adaptation. This suggests that interlimb transfer may be occurring at a level where only very general information is transferable-for example the perceived direction of a visual-motor displacement (Malfait and Ostry 2004).

Unimanual dual adaptation to opposing novel environments has been shown to be either very slow (Osu et al. 2004; Wada et al. 2003) or even impossible (Krakauer et al. 1999; Karniel and Mussa-Ivaldi 2002). In contrast Wada et al. (2003) have shown that when the opposing environments can be easily discriminated and the contextual information is clear and distinct, dual adaptation is possible. Hwang et al. (2006) showed that optimal performance within a dual adaptation task occurs when the subject has visual and proprioceptive cues. They also found that proprioceptive cues have a significantly larger influence on performance than visual cues, although dual adaptation was seen within both situations.

As interlimb transfer may be occurring at a very abstract level of movement representation (Malfait and Ostry 2004) it is unknown whether the more complex representation involved within dual adaptation would transfer across limbs. However the development of an effector independent visual model does seem to take place during dual adaptation to opposing dynamic forces, and is proposed to be a prerequisite for interlimb transfer (Hwang et al. 2006).

This study tested whether adaptation to opposing dynamic viscous forces with one limb would improve the initial performance of the other limb. Our aim was to initially extend the findings of previous interlimb transfer studies by indicating whether a more complex task involving dual adaptation to opposing states could be transferred.

The results will also indicate whether transfer occurs across an extrinsic spatial or intrinsic joint based coordinate system. On the basis of Hwang et al.'s (2006) predictions, transfer should always be observed across an extrinsic coordinate system as it is based on the explicit visual model. However Wang and Sainburg (2004a) found transfer across an intrinsic coordinate system suggesting that the coordinate system used to represent movement within the explicit visual model may not be consistent.

To test these ideas subjects made movements to targets with either the left or right limb, with the type of viscous force (either assistive or resistive) experienced on a specific trial dependent on the location of the target. Then the other limb was exposed to the two forces, with each force associated with targets in either similar spatial locations, which can be described as an extrinsic coordinative system, or mirror symmetrically similar locations, which can be described as an intrinsic, joint based, coordinative system (Wang and Sainburg 2004a).

\section{Method}

Subjects

Twenty-five healthy subjects (13 male and 12 female) with a mean age of $25( \pm 4)$ took part. They were all right handed and had normal or corrected to normal vision. Righthandedness was assessed using the ten-item version of the Edinburgh inventory (Oldfield 1971) (mean $=68$, decile R.3). All subjects were recruited from the university community and were paid to participate whilst providing informed consent. This study was approved by the school ethics committee and therefore was performed in accordance with the ethical standards laid down in the 1964 Declaration of Helsinki.

Materials and procedure

Subjects sat facing two-mirror symmetric vBOT motor driven manipulanda (Bays and Wolpert 2006) with a computer screen approximately a metre in front of them at eye level. The subjects grasped the handle of the vBOT directly in front of the arm in use; handle position was displayed as a marker on the screen. Each arm was supported at the elbow with a sling and the subjects wore goggles with a horizontal blinker ensuring their arms were not visible. In order to maintain both extrinsic and intrinsic dissociation of targets, opposing viscous forces were used as they would not distort the movement direction but would still influence hand trajectory (Wada et al. 2003). The manipulanda generated the viscous force as shown in Eq. (1).

$\tau=c \times v$

where $\tau$ is product of the manipulandum velocity $(v)$ and a viscous constant $(c)$, specified in $\mathrm{N} /(\mathrm{cm} / \mathrm{s})$. The viscous constants used were -0.4 for a resistive force-field and 0.12 for an assistive force-field. Our aim was to primarily dissociate the movement towards each target through different extrinsic workspace locations. However, the target locations were also based on the preferred angular direction of a specific muscle (Thoroughman and Shadmehr 1999). Computer generated visual targets appeared on the screen randomly in one of four positions (Fig. 1). These positions were based on Thoroughman and Shadmehr's (1999) estimations of the directional bias for the 


\section{BASELINE(32)}

TRAIN (48)

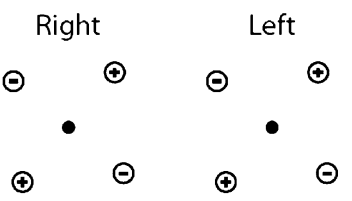

Group RL_ext

Right $\rightarrow$ Left

Extrinsic

\section{Group LR_ext}

Left $\rightarrow$ Right

Extrinsic

\begin{tabular}{|c|c|c|c|c|c|}
\hline \multirow[b]{2}{*}{ Group RL_int } & & \multicolumn{2}{|c|}{ Right } & \multicolumn{2}{|c|}{ Left } \\
\hline & & (๑) & $\Theta$ & $\Theta$ & $\oplus$ \\
\hline Right $\rightarrow$ Left & Null & & & & \\
\hline \multirow[t]{2}{*}{ Intrinsic } & & $\Theta$ & $\odot$ & $\odot$ & $\Theta$ \\
\hline & & \multicolumn{2}{|c|}{ Left } & \multicolumn{2}{|c|}{ Right } \\
\hline Group LR_int & & ( & $\Theta$ & $\Theta$ & $\odot$ \\
\hline Left $\rightarrow$ Right & Null & & & & \\
\hline Intrinsic & & $\Theta$ & $\oplus$ & (†) & \\
\hline
\end{tabular}

Fig. 1 The four main experimental groups. The numbers in brackets indicate the amount of movements to each target. The baseline involved a set with the left and right arms experiencing the task within a null environment. The figure indicates exact target locations. For the extrinsic groups the targets have similar spatial locations for the train and test phases (e.g., top row). For the intrinsic groups the targets have similar joint angle positions for the train and test phases (e.g., bottom row). For each target: - resistive force, + assistive force. These indicate the force applied to the target within that quadrant

biceps, triceps, anterior deltoid and posterior deltoid. Each target represented the direction in which maximal activation occurred for one of these particular muscles. Hence the targets were proposed to be separated by both visual location (extrinsic) and by the activation of a primary muscle (intrinsic). The subjects were required to move from the starting position towards the visible target with a smooth, straight trajectory and a clear end-point. Once they had began to move, the marker indicating their hand position disappeared, providing no online visual feedback. Once the movement fell below a velocity threshold an end error marker appeared; this was red, green or yellow. Red indicated that their movement was too slow; green too fast and yellow was within the required time range of 500-600 ms. The marker then reappeared allowing the subject to move back to the starting position in the centre of the screen with online feedback, but with no forces applied to the manipulanda.
Experimental protocol

There were five groups (Fig. 1). All conditions involved a baseline phase where each arm moved to each 4 targets 32 times (128) while experiencing a null force. The training phase involved moving to each target 48 times (192) and the test phase involved moving to each target 26 times (104).

For group RL_ext and LR_ext, during the training and test phases the forces occurred in similar spatial locations (extrinsic coordinate system) (Fig. 1). Group RL_ext tested dominant $\rightarrow$ non-dominant arm transfer, while group LR_ext tested non-dominant $\rightarrow$ dominant arm transfer. For group RL_int and LR_int the training and test phases involved forces occurring in opposing spatial locations but in similar joint directions (intrinsic coordinate system) (Fig. 1). Group RL_int tested for dominant $\rightarrow$ non-dominant arm transfer and group LR_int tested non-dominant $\rightarrow$ dominant arm transfer.

For groups with forces in similar extrinsic coordinates the target positions remained the same across the training and test phases. Based on Thoroughman and Shadmehr's (1999) estimations group RL_ext had target positions of $40^{\circ}, 130^{\circ}, 220^{\circ}$ and $310^{\circ}$ from the positive $x$-axis and group LR_ext had positions of $50^{\circ}, 140^{\circ}, 230^{\circ}$ and $320^{\circ}$. For groups with forces in similar intrinsic coordinates the target positions were adjusted across the training and test phase. Group RL_int began with positions of $40^{\circ}, 130^{\circ}$, $220^{\circ}$ and $310^{\circ}$. The targets would then move $10^{\circ}$ in a counter-clockwise direction to $50^{\circ}, 140^{\circ}, 230^{\circ}$ and $320^{\circ}$ in the test phase. The opposite occurred for the LR_int group (Fig. 1).

As the training phase in group LR_ext and test phase in group RL_int involved slightly different target locations for left hand movements, a final group L_control was required as a naïve performance comparison for group RL_int. L_control only involved a baseline and training phase and the training phase had similar target locations to the left hand within RL_int.

Analysis

Hand positions and speeds were sampled at $60 \mathrm{~Hz}$ with movements exceeding $2 \mathrm{~s}$ being discarded $(<1 \%)$. Speeds were then resampled to a uniform 200 samples for each movement. In order to compare hand trajectories a technique developed by Shadmehr and Mussa-Ivaldi (1994) was used which quantified a measure of correlation between two sampled vector fields. In order to calculate a 'typical' hand trajectory the resampled speed profiles of the last ten movements towards each target within the baseline 
period were averaged for each subject. These movements were required to have durations between 450 and $650 \mathrm{~ms}$. Individual resampled movements during the train and test phases were then correlated with the 'typical' speed profile calculated for the relevant target. This produced a correlation coefficient $(r)$ indicating the amount of similarity between the speed profiles. The coefficient $(r)$ values were then converted into $z$-scores using Fisher's transformation (Eq. 2) (Fisher 1915, 1921). By using this transformation $r$ is converted into a $z$ value, which is normally distributed, and so can be used in parametric tests

$z_{(n)}=1 / 2 \log \left[1+r_{(n)} / 1-r_{(n)}\right]$.

Epochs were created by averaging $z$ across three trials; for each target location there were 16 epochs for the training phase and 8 epochs for the test phase. As a result only the initial eight epochs within the training phase was used for comparison. As no significant differences were found between movements towards the two assistive and, separately, the two resistive targets these pairs were combined for all analysis.

Separate repeated measures ANOVAs were conducted on the non-dominant $\rightarrow$ dominant (train. RL_ext, test. LR_ext and test. LR_int) and dominant $\rightarrow$ non-dominant (train. LR_ext, test. RL_ext and test. RL_int) comparisons with group (3) as a between-group factor and force (resistive, assistive) and epoch (8) as within-group factors. Bonferroni post-hoc tests were performed on all significant between-group comparisons.

\section{Results}

\section{Summary}

During the baseline phase normal bell-shaped speed profiles were produced. When initially exposed to the velocity dependent viscous forces the hand trajectories were distorted. The assistive force caused maximal speed to both increase and take place earlier in the movement. A second corrective peak also appeared. The resistive force caused a decrease in maximal speed and a slower deceleration phase. The shape and size of the speed profile was affected more by the assistive than resistive force. However, by the end of the training phase the subject's profiles move towards 'typical' baseline performance.

Figure 2 shows the transformed correlation coefficient values $(z)$ averaged across groups. A high $z$ value corresponded to a strong correlation between the speed profiles of a movement within a force and a 'typical' movement within baseline. Using repeated measures ANOVAs (3 groups $\times 2$ forces $\times 8$ epochs) two sets of comparisons were made: non-dominant $\rightarrow$ dominant (ND-D) (train. RL_ext, test. LR_ext and test. LR_int) and dominant $\rightarrow$ non-dominant (D-ND) (train. LR_ext, test. RL_ext and test. RL_int). For both comparisons there was a main effect for epoch $\left[F_{(7,189)} \geq 5.296, P \leq 0.0005\right]$ and force $\left[F_{(1,27)} \geq 112.978, P \leq 0.0005\right]$. However there was only a main effect of condition for the (D-ND) comparison $\left[F_{(1,27)}=18.502, P=0.0005\right]$.

\section{Training}

Figure $2 \mathrm{a}, \mathrm{b}$ shows the training phase for the four experimental groups. In order to assess the level of final adaptation, a comparison was made across groups using the final three epochs (14-16). For the resistive force the main effect of epoch and group on final adaptation level were not significant. For the assistive force the main effect of epoch was not significant, however, there was a main effect for group $\left[F_{(3,36)}=2.924, P<0.047\right]$. A bonferroni post-hoc test failed to find any significant differences between the groups $(P \geq 0.202)$. The non-significant effect for epoch indicates that for both assistive and resistive forces a plateau of performance had been reached before the last three epochs of the training phases. For the resistive force a similar level of performance was observed across groups, however, for the assistive force there was an observable difference between hands. Figure $2 b$ clearly shows the right hand groups (RL_int and RL_ext) produced higher $z$ scores in comparison to the left hand groups (LR_int and LR_ext). However, when assessing interlimb transfer only data from the left or right hand were compared. This means that for all comparisons an equivalent level of performance had been reached in the relevant, compared, groups.

The two main groups for assessing naive performance without opposite limb training were RL_ext train and LR_ext train. Figure 2c-f shows that for both there is an increase in the $z$ score from epoch one to eight with the main effect of epoch confirming this $(P \leq 0.0005)$. The improvement ranged from 14 to $24 \%$ for the resistive force, and $37-41 \%$ for the assistive force. This indicates that adaptation to the opposing dynamic forces took place for the right (RL_ext train) and left (LR_ext train) arms during naïve performance.

It is also clear that the assistive force caused a larger decrement in performance in comparison to the resistive force with the main effect of force $(P \leq 0.0005)$ confirming this (Fig. 2 note the difference in vertical scale for the upper and lower graphs). Within epoch one the initial $z$ scores were $0.46-0.50$ for the assistive force and 1.04-1.18 for the resistive force. The lower $z$ scores within an assistive force indicate that there was a larger deviation from a 'typical' baseline velocity profile. 
Fig. 2 The average

transformed correlation coefficient $(z)$ for movements within each group in a force field as compared to movements in a null field, as a function of practise epochs in the force field $( \pm \mathrm{SE})$. Training phase across all four conditions with either a resistive (a) or assistive force (b). c Non-dominant to dominant across extrinsically (LR_ext) and intrinsically (LR_int) similar target positions with resistive forces. d Nondominant to dominant across extrinsically (RL_ext) and intrinsically (RL_int) similar target positions with assistive forces. e Dominant to nondominant across extrinsically (RL_ext) and intrinsically (RL_int) similar target positions with resistive forces. $\mathbf{f}$ Dominant to non-dominant across extrinsically (RL_ext) and intrinsically (RL_int) similar target positions with assistive forces
RESISTIVE
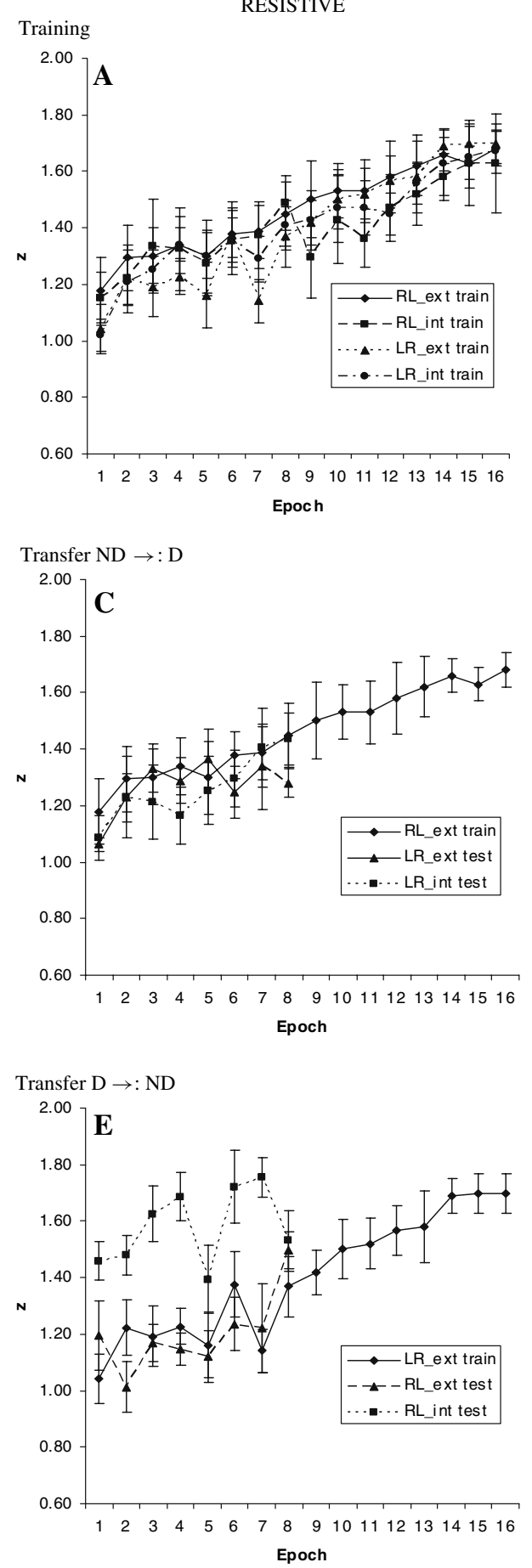

ASSISTIVE
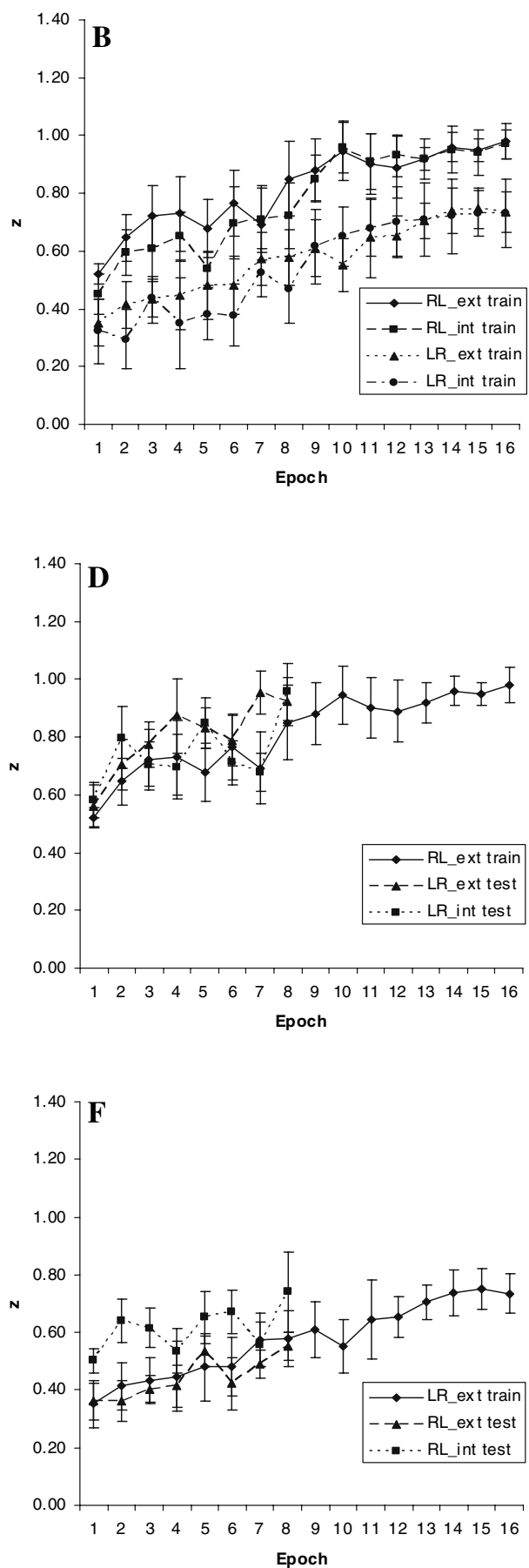

\section{Interlimb transfer}

In order to test for interlimb transfer the naïve performance in the training phase was compared with the performance, after opposite limb training, in the test phase. Figure 2 shows the comparisons made. As mentioned in "Method", the test phase in group RL_int was compared with the training phase within group L_control. However, as there was no significant differences between the training phases of group RL_int and L_control $(P=0.211)$, even though they had slightly different target positions, only RL_int is shown in Fig. 2.

For the (ND-D) comparison Fig. 2c, d shows that for both forces there is an increase in $z$ from epoch one to eight however this is consistent across groups. This observation is supported by the main effect of epoch $(P=0.0005)$ and 
the non-significant main effect of group $(P=0.889)$ and interaction (epoch $\times$ group) $(P=0.576)$. This indicates that prior training with the non-dominant left arm did not improve the subsequent performance of the dominant right arm.

For the (D-ND) comparison, Fig. 2e, f shows that there is a noticeable difference between RL_int test and the other two groups (LR_ext train and RL_ext test). This is confirmed by the significant main effect of group $(P=0.0005)$. Bonferroni post-hoc comparisons showed a significant difference between RL_int test and the other two groups LR_ext train $(P=0.0005)$ and RL_ext test $(P=0.0005)$. This suggests that for both forces prior training with the dominant right arm improved the performance of the non-dominant left arm. This interlimb transfer only occurred when the forces were similar across an intrinsic coordinate system as RL_ext test was no different from LR_ext train $(P=1)$.

\section{Discussion}

\section{Summary}

The aim of this study was to evaluate whether concurrent adaptation to opposing viscous forces with one limb would improve the initial performance of the other. The results indicate that interlimb transfer occurred from the dominant to the non-dominant limb across an intrinsic coordinative system.

\section{Training}

It has been proposed that adaptation to opposing forces can be measured as an increased correlation coefficient between the speed profiles of individual movements made within, a force, and a 'typical velocity profile' within a null field (Shadmehr and Mussa-Ivaldi 1994; Wada et al. 2003). Within all training groups this value increases from epoch one to eight. Importantly, for all the training groups there was a significant improvement suggesting dual adaptation to the opposing forces had occurred.

There is a noticeable difference between the correlation coefficients seen in movements within an assistive and resistive force. The movements within the resistive force had transformed coefficients of 1-1.7 whilst movements within the assistive force had lower values between 0.3 and 1. This suggests that the movement trajectory was distorted more by the assistive than the resistive force. Since the forces were velocity dependent, this difference can be explained by positive-feedback. The assistive force caused an increase in the velocity of the movement, which in turn resulted in the force becoming even stronger. The opposite effect is seen with the resistive force where an increase in force causes the movement speed to slow, (i.e.,) a negative feedback loop. Hence the small improvements in adaptation seen within the resistive force may be attributable to the negative feedback effect, such that the initial deviations in movement trajectory were small.

Within the training phase there were differences between the final adaptation levels, specifically in the assistive force condition where there was a clear difference between the groups which trained with either the left or right arm. Importantly however, when a comparison was made between groups their final adaptation values were always similar for the training phase. Moreover, as no significant differences were found between the last three epochs of the training phase it suggests all groups had reached a plateau in performance.

Interlimb transfer

The interlimb transfer we observed from the dominant to the non-dominant limb is in agreement with previous work with adaptation to a single dynamic force (CriscimagnaHemminger et al. 2003; Dizio and Lackner 1995; Malfait and Ostry 2004; Wang and Sainburg 2004a). Interlimb transfer supports the idea that motor adaptation is not completely effector specific; however asymmetric transfer suggests effector dependent components (Vangheluwe et al. 2005; Wang and Sainburg 2004b).

Previous studies have reported transfer across different coordinate systems. Criscimagna-Hemminger et al. (2003) and Malfait and Ostry (2004) used a velocity dependent force and found transfer across an extrinsic coordinate system. In contrast Wang and Sainburg (2004a) used a constant inertial force and found transfer across an intrinsic coordinative system. As we used velocity dependent viscous forces it might be expected that our results would be similar to those of Criscimagna-Hemminger et al. (2003) and Malfait and Ostry (2004); instead we found transfer across an intrinsic coordinate system.

The relationship between different types of forces, sensory information and the resulting pattern of interlimb generalization remains unclear (Wang and Sainburg 2004a). However Hwang et al. (2006) recently proposed that interlimb transfer is a consequence of an effector independent internal model which is predominately based vision. They believe that transfer should only occur in extrinsic coordinates and is highly dependent on the subject becoming explicitly aware of the novel environment.

We provide evidence that interlimb transfer can occur across intrinsic coordinates supporting previous work by Wang and Sainburg (2004a). They conclude that the 
pattern of interlimb transfer may not have followed an extrinsic coordinate system due to their force perturbation being inertial and so changing the type of proprioceptive information the subjects received. However according to Hwang et al. (2006) the type of proprioceptive information should not influence interlimb transfer, as it has little contribution to the explicit visual model. The present study shows that transfer across intrinsic coordinates can occur with viscous velocity dependent forces. This suggests that a factor other than the type of dynamic force is determining the pattern of interlimb transfer.

Within our experimental set-up subjects received only terminal vision and the forces they experienced resulted in minimal observable error in the end position of their movement. This would suggest that they lacked visual cues regarding the forces they were experiencing. However, it is very likely that the subjects were still aware of these perturbations, as they were clearly detectable. We propose that explicit awareness of the perturbation facilitates interlimb transfer and awareness is four times more dependent on visual information than proprioceptive information (Malfait and Ostry 2004; Hwang et al. 2006). However, as the visual errors are both small and do not directly identify the two force conditions, while the forces are clearly proprioceptively detectable, interlimb transfer occurs in intrinsic coordinates. Further investigation is required to understand how such intrinsic interlimb transfer could be accounted for by the model proposed by Hwang et al. (2006).

It has also been suggested that the general movement goal (Vangheluwe et al. 2005) or perceived direction of a visual-motor displacement (Malfait and Ostry 2004) constitutes the higher level of movement representation, which allows interlimb transfer. The present results suggest that at this level opposing motor states can be concurrently represented and transferred in an effector specific manner. This implies that the information available at this level can be more intricate than a general movement goal or a single perceived directional error.

In summary, these results suggest that two opposing motor states can be concurrently transferred across limbs. The results are specific to one direction (dominant $\rightarrow$ nondominant) and one coordinate system (intrinsic). We propose that this interlimb transfer is a consequence of a higher level of movement representation, which is effector independent. Interestingly, negative interference was not seen when the targets were opposed across an extrinsic coordinate system, supporting previous studies which suggest that if the information to be transferred is not useful then the link between the limbs is not made (Wang and Sainburg 2004b).

\section{References}

Bays PM, Wolpert DM (2006) Actions and consequences in bimanual interaction are represented in different coordinate systems. J Neurosci 26(26):7121-7126

Criscimagna-Hemminger SE, Donchin O, Gazzaniga MS, Shadmehr R (2003) Learned dynamics of reaching movements generalize from dominant to nondominant arm. J Neurophysiol 89:168-176

Dizio P, Lackner JR (1995) Motor adaptation to Coriolis-force perturbations of reaching movements-end-point but not trajectory adaptation transfers to the nonexposed arm. J Neurophysiol 74:1787-1792

Fisher RA (1915) Frequency distribution of the values of the correlation coefficient in samples of an independently large population. Biometrika 10:507-521

Fisher RA (1921) On the 'probable error' of a coefficient of correlation deduced from a small sample. Metron 1:3-32

Hwang EJ, Smith MA, Shadmehr R (2006) Dissociable effects of the implicit and explicit memory systems on learning control of reaching. Exp Brain Res 173:425-437

Karniel A, Mussa-Ivaldi FA (2002) Does the motor control system use multiple models and context switching to cope with a variable environment? Exp Brain Res 143:520-524

Krakauer JW, Ghilardi M-F, Ghez C (1999) Independent learning of internal models for kinematic and dynamic control of reaching. Nat Neurosci 2:1026-1031

Malfait N, Ostry DJ (2004) Is interlimb transfer of force-field adaptation a cognitive response to the sudden introduction of load? J Neurosci 24:8084-8089

Oldfield RC (1971) The assessment and analysis of handeness: the Edinburgh inventory. Neuropsychologia 9:97-113

Osu R, Hirai S, Yoshioka T, Kawato M (2004) Random presentation enables subjects to adapt to two opposing forces on the hand. Nat Neurosci 7(2):111-112

Sainburg RL, Wang JS (2002) Interlimb transfer of visuomotor rotations: independence of direction and final position information. Exp Brain Res 145:437-447

Shadmehr R, Mussa-Ivaldi FA (1994) Adaptive representation of dynamics during learning of a motor task. J Neurosci 14:3208-3224

Thoroughman KA, Shadmehr R (1999) Electromyographic correlates of learning an internal model of reaching movements. J Neurosci 19:8573-8588

Vangheluwe S, Wenderoth N, Swinnen SP (2005) Learning and transfer of an ipsilateral coordination task: evidence for a duallayer movement representation. J Cogn Neurosci 17:1460-1470

Wada Y, Kawabata Y, Kotosaka S, Yamamoto K, Kitazawa S, Kawato M (2003) Acquisition and contextual switching of multiple internal models for different viscous force fields. Neurosci Res 46:319-331

Wang JS, Sainburg RL (2003) Mechanisms underlying interlimb transfer of visuomotor rotations. Exp Brain Res 149:520-526

Wang JS, Sainburg RL (2004a) Interlimb transfer of novel inertial dynamics is asymmetrical. J Neurophysiol 92:349-360

Wang JS, Sainburg RL (2004b) Limitations in interlimb transfer of visuomotor rotations. Exp Brain Res 155:1-8 